\title{
Baryon-baryon interactions from Effective Field Theory
}

\author{
J. Haidenbauer ${ }^{1, a}$ \\ Institute for Advanced Simulation and Jülich Centre for Hadron Physics, Forschungszentrum Jülich GmbH, D-52425 \\ Jülich, Germany
}

\begin{abstract}
I report on recent progress in the description of baryon-baryon systems within chiral effective field theory. In particular, results for the hyperon-nucleon and hyperon-hyperon interactions as well as for strangeness $S=-3$ to -4 baryon-baryon systems, obtained to leading order are discussed. Preliminary results for the hyperon-nucleon interaction to next-to-leading order are presented.
\end{abstract}

\section{Introduction}

Chiral effective field theory (EFT) as proposed in the pioneering works of Weinberg $[1,2]$ is a powerful tool for the derivation of nuclear forces. In this scheme there is an underlying power counting which allows to improve calculations systematically by going to higher orders in a perturbative expansion. In addition, it is possible to derive twoand corresponding three-nucleon forces as well as external current operators in a consistent way. For reviews we refer the reader to Refs. [3-5].

Over the last decade or so it has been demonstrated that the nucleon-nucleon $(N N)$ interaction can be described to a high precision within the chiral EFT approach [6,7]. Following the original suggestion of Steven Weinberg, in these works the power counting is applied to the $N N$ potential rather than to the reaction amplitude. The latter is then obtained from solving a regularized Lippmann-Schwinger equation for the derived interaction potential. The $N N$ potential contains pion-exchanges and a series of contact interactions with an increasing number of derivatives to parameterize the shorter ranged part of the $N N$ force.

Recently, also hadronic systems involving the strange baryons $\Lambda$ and $\Sigma$ were investigated within EFT. For example, hyperon and nucleon mass shifts in nuclear matter have been studied in [8] using chiral perturbation theory. These authors used a chiral interaction containing fourbaryon contact terms and pseudoscalar-meson exchanges. Furthermore, the hypertriton and $\Lambda d$ scattering were investigated in the framework of an EFT with contact interactions [9]. Korpa et al. [10] performed a next-to-leading order (NLO) EFT analysis of $Y N$ scattering and hyperon mass shifts in nuclear matter. Their tree-level amplitude contains four-baryon contact terms; pseudoscalar-meson exchanges were not considered explicitly, but SU(3) $)_{f}$ breaking by meson masses was modeled by incorporating dimension two terms coming from one-pion exchange. The full scattering amplitude was calculated using the KaplanSavage-Wise resummation scheme [11]. Some aspects of

\footnotetext{
a e-mail: j.haidenbauer@fz-juelich.de
}

strong $\Lambda N$ scattering in effective field theory and its relation to various formulations of lattice QCD are discussed in [12].

In the present contribution I focus on recent investigations by the group in Jülich on the baryon-baryon interaction involving strange baryons, performed within chiral EFT [13-16]. In these works the same scheme as applied in Ref. [7] to the $N N$ interaction is adopted. To begin with I discuss briefly our initial results for the (strangeness $S=-1) \Lambda N$ and $\Sigma N$ channels [13]. Then I report on the extension of that study to the strangeness $S=-2$ sector, i.e. to the $\Lambda \Lambda, \Sigma \Sigma$, and cascade-nucleon $(\Xi N)$ interactions [14], and also to systems with $S=-3$ and -4 [15].

At leading order (LO) in the power counting, as considered in the aforementioned investigations [13-15], the baryon-baryon potentials involving strange baryons consist of four-baryon contact terms without derivatives and of one-pseudoscalar-meson exchanges, analogous to the $N N$ potential of [7]. The potentials are derived using constraints from SU(3) flavor symmetry. At next-to-leading order (NLO) one gets contributions from two-pseudoscalarmeson exchange diagrams and from four-baryon contact terms with two derivatives [7]. The extension of our study to NLO is in progress [16] and a first glimpse on our (still preliminary) results for the $\Lambda N$ and $\Sigma N$ interactions will be given towards the end of my presentation.

The paper is structured as follows: In Sect. 2 a short overview of the chiral EFT approach is provided. In Sect. 3 our LO results for the $S=-1(\Lambda N, \Sigma N)$ and $S=-2$ $(\Lambda \Lambda$, etc.) systems are briefly reviewed. Recently we have also considered the (coupled) $\Xi \Lambda-\Xi \Sigma$ system and the $\Xi \Xi$ channel within chiral EFT. Corresponding predictions are presented in Sect. 4 and discussed in more detail. Finally, preliminary results of a not yet complete NLO calculation for the $\Lambda N$ and $\Sigma N$ interactions are reported in Sect. 5. The paper ends with a short summary. 
Table 1. Various LO baryon-baryon contact potentials for the ${ }^{1} S_{0}$ and ${ }^{3} S_{1}$ partial waves in the isospin basis. $C^{27}$ etc. refers to the corresponding $\mathrm{SU}(3)_{\mathrm{f}}$ irreducible representation $[18,19]$.

\begin{tabular}{|c|c|c|c|c|c|}
\hline & Channel & Isospin & $C_{1 S 0}$ & Isospin & $C_{3 S 1}$ \\
\hline$S=0$ & $N N \rightarrow N N$ & 1 & $C^{27}$ & 0 & $C^{10^{*}}$ \\
\hline$S=-1$ & $\begin{array}{l}\Lambda N \rightarrow \Lambda N \\
\Lambda N \rightarrow \Sigma N \\
\Sigma N \rightarrow \Sigma N \\
\Sigma N \rightarrow \Sigma N\end{array}$ & $\begin{array}{l}\frac{1}{2} \\
\frac{1}{2} \\
\frac{1}{2} \\
\frac{3}{2}\end{array}$ & $\begin{array}{l}\frac{1}{10}\left(9 C^{27}+C^{8_{s}}\right) \\
\frac{3}{10}\left(-C^{27}+C^{8_{s}}\right) \\
\frac{1}{10}\left(C^{27}+9 C^{8_{s}}\right) \\
C^{27}\end{array}$ & $\begin{array}{l}\frac{1}{2} \\
\frac{1}{2} \\
\frac{1}{2} \\
\frac{3}{2} \\
\end{array}$ & $\begin{array}{l}\frac{1}{2}\left(C^{8_{a}}+C^{10^{*}}\right) \\
\frac{1}{2}\left(-C^{8_{a}}+C^{10^{*}}\right) \\
\frac{1}{2}\left(C^{8_{a}}+C^{10^{*}}\right) \\
C^{10}\end{array}$ \\
\hline$S=-2$ & $\begin{array}{l}\Lambda \Lambda \rightarrow \Lambda \Lambda \\
\Lambda \Lambda \rightarrow \Xi N \\
\Lambda \Lambda \rightarrow \Sigma \Sigma \\
\Xi N \rightarrow \Xi N \\
\Xi N \rightarrow \Sigma \Sigma \\
\Sigma \Sigma \rightarrow \Sigma \Sigma \\
\Xi N \rightarrow \Xi N \\
\Xi N \rightarrow \Sigma \Lambda \\
\Sigma \Lambda \rightarrow \Sigma \Lambda \\
\Sigma \Lambda \rightarrow \Sigma \Sigma \\
\Sigma \Sigma \rightarrow \Sigma \Sigma\end{array}$ & $\begin{array}{l}0 \\
0 \\
0 \\
0 \\
0 \\
0 \\
1 \\
1 \\
1 \\
2 \\
2\end{array}$ & $\begin{array}{l}\frac{1}{40}\left(27 C^{27}+8 C^{8_{s}}+5 C^{1}\right) \\
\frac{-1}{40}\left(18 C^{27}-8 C^{8_{s}}-10 C^{1}\right) \\
\frac{\sqrt{3}}{40}\left(-3 C^{27}+8 C^{8_{s}}-5 C^{1}\right) \\
\frac{1}{40}\left(12 C^{27}+8 C^{8_{s}}+20 C^{1}\right) \\
\frac{\sqrt{3}}{40}\left(2 C^{27}+8 C^{8_{s}}-10 C^{1}\right) \\
\frac{1}{40}\left(C^{27}+24 C^{8_{s}}+15 C^{1}\right) \\
\frac{1}{5}\left(2 C^{27}+3 C^{8_{s}}\right) \\
\frac{\sqrt{6}}{5}\left(C^{27}-C^{8_{s}}\right) \\
\frac{1}{5}\left(3 C^{27}+2 C^{8_{s}}\right) \\
C^{27}\end{array}$ & $\begin{array}{l}1 \\
1 \\
1 \\
1 \\
1 \\
1\end{array}$ & $\begin{array}{l}C^{8_{a}} \\
\frac{\sqrt{2}}{6}\left(C^{10}+C^{10^{*}}-2 C^{8_{a}}\right) \\
\frac{1}{6}\left(C^{10}+C^{10^{*}}+4 C^{8_{a}}\right) \\
\frac{1}{3}\left(C^{10}+C^{10^{*}}+C^{8_{a}}\right) \\
\frac{\sqrt{6}}{6}\left(C^{10}-C^{10^{*}}\right) \\
\frac{1}{2}\left(C^{10}+C^{10^{*}}\right) \\
\frac{\sqrt{3}}{6}\left(C^{10}-C^{10^{*}}\right)\end{array}$ \\
\hline$S=-3$ & $\begin{array}{l}\Xi \Lambda \rightarrow \Xi \Lambda \\
\Xi \Lambda \rightarrow \Xi \Sigma \\
\Xi \Sigma \rightarrow \Xi \Sigma \\
\Xi \Sigma \rightarrow \Xi \Sigma\end{array}$ & $\begin{array}{l}\frac{1}{2} \\
\frac{1}{2} \\
\frac{1}{2} \\
\frac{3}{2} \\
\end{array}$ & $\begin{array}{l}\frac{1}{10}\left(9 C^{27}+C^{8_{s}}\right) \\
\frac{3}{10}\left(-C^{27}+C^{8_{s}}\right) \\
\frac{1}{10}\left(C^{27}+9 C^{8_{s}}\right) \\
C^{27}\end{array}$ & $\begin{array}{l}\frac{1}{2} \\
\frac{1}{2} \\
\frac{1}{2} \\
\frac{3}{2} \\
\end{array}$ & $\begin{array}{l}\frac{1}{2}\left(C^{8 a}+C^{10}\right) \\
\frac{1}{2}\left(-C^{8_{a}}+C^{10}\right) \\
\frac{1}{2}\left(C^{8 a}+C^{10}\right) \\
C^{10^{*}} \\
\end{array}$ \\
\hline$S=-4$ & $\Xi \Xi \rightarrow \Xi \Xi$ & 1 & $C^{27}$ & 0 & $C^{10}$ \\
\hline
\end{tabular}

\section{Formalism}

Details on the derivation of the chiral baryon-baryon potentials for the strangeness sector at LO using the Weinberg power counting can be found in Refs. [13], [15] and [17].

The LO potential consists of four-baryon contact terms without derivatives and of one-pseudoscalar-meson exchanges.

The LO SU(3) invariant contact terms for the octet baryon-baryon interactions that are Hermitian and invariant under Lorentz transformations follow from the Lagrangians

$$
\begin{aligned}
\mathcal{L}^{1} & =C_{i}^{1}\left\langle\bar{B}_{a} \bar{B}_{b}\left(\Gamma_{i} B\right)_{b}\left(\Gamma_{i} B\right)_{a}\right\rangle, \\
\mathcal{L}^{2} & =C_{i}^{2}\left\langle\bar{B}_{a}\left(\Gamma_{i} B\right)_{a} \bar{B}_{b}\left(\Gamma_{i} B\right)_{b}\right\rangle, \\
\mathcal{L}^{3} & =C_{i}^{3}\left\langle\bar{B}_{a}\left(\Gamma_{i} B\right)_{a}\right\rangle\left\langle\bar{B}_{b}\left(\Gamma_{i} B\right)_{b}\right\rangle .
\end{aligned}
$$

Here $a$ and $b$ denote the Dirac indices of the particles, $B$ is the irreducible octet (matrix) representation of $\mathrm{SU}(3)_{\mathrm{f}}$, and the $\Gamma_{i}$ are the usual elements of the Clifford algebra [13].

As described in Ref. [13], to LO the Lagrangians in Eq. (1) give rise to only six independent low-energy coefficients (LECs) $-C_{S}^{1}, C_{T}^{1}, C_{S}^{2}, C_{T}^{2}, C_{S}^{3}$ and $C_{T}^{3}-$ due to $\mathrm{SU}(3)_{\mathrm{f}}$ constraints. They need to be determined by a fit to experimental data. The subscripts $S$ and $T$ refer to the central and spin-spin parts of the potential, respectively. The spin- and momentum structure of the potentials to LO is given by

$$
V^{(0)}=C_{S}+C_{T}\left(\sigma_{1} \cdot \sigma_{2}\right) .
$$

It is convenient to re-express the baryon-baryon potentials in terms of the $\mathrm{SU}(3)_{\mathrm{f}}$ irreducible representations, see e.g. $[18,19]$. Then the contact interaction is given by

$$
V^{(0)}=\frac{1}{4}\left(1-\sigma_{1} \cdot \sigma_{2}\right) C_{1 S 0}+\frac{1}{4}\left(3+\sigma_{1} \cdot \sigma_{2}\right) C_{3 S 1},
$$

and the constraints imposed by the assumed $\mathrm{SU}(3)_{\mathrm{f}}$ symmetry on the interactions in the various baryon-baryon channels for the ${ }^{1} S_{0}$ and ${ }^{3} S_{1}$ partial waves can be readily read off from Table 1.

The lowest order $\mathrm{SU}(3)_{\mathrm{f}}$ invariant pseudoscalarmeson-baryon interaction Lagrangian with the appropriate symmetries was discussed in [13]. In the isospin basis it reads

$$
\begin{aligned}
\mathcal{L}= & -f_{N N \pi} \bar{N} \gamma^{\mu} \gamma_{5} \tau N \cdot \partial_{\mu} \pi+i f_{\Sigma \Sigma \pi} \bar{\Sigma} \gamma^{\mu} \gamma_{5} \times \Sigma \cdot \partial_{\mu} \pi \\
& -f_{\Lambda \Sigma \pi}\left[\bar{\Lambda} \gamma^{\mu} \gamma_{5} \Sigma+\bar{\Sigma} \gamma^{\mu} \gamma_{5} \Lambda\right] \cdot \partial_{\mu} \pi \\
& -f_{\Xi \Xi \pi} \bar{\Xi} \gamma^{\mu} \gamma_{5} \tau \Xi \cdot \partial_{\mu} \pi \\
& -f_{\Lambda N K}\left[\bar{N} \gamma^{\mu} \gamma_{5} \Lambda \partial_{\mu} K+\bar{\Lambda} \gamma^{\mu} \gamma_{5} N \partial_{\mu} K^{\dagger}\right] \\
& -f_{\Xi \Lambda K}\left[\bar{\Xi} \gamma^{\mu} \gamma_{5} \Lambda \partial_{\mu} K_{c}+\bar{\Lambda} \gamma^{\mu} \gamma_{5} \Xi \partial_{\mu} K_{c}^{\dagger}\right] \\
& -f_{\Sigma N K}\left[\bar{\Sigma} \cdot \gamma^{\mu} \gamma_{5} \partial_{\mu} K^{\dagger} \tau N+\bar{N} \gamma^{\mu} \gamma_{5} \tau \partial_{\mu} K \cdot \Sigma\right]
\end{aligned}
$$


$19^{\text {th }}$ International IUPAP Conference on Few-Body Problems in Physics

$$
\begin{aligned}
& -f_{\Xi \Sigma K}\left[\bar{\Sigma} \cdot \gamma^{\mu} \gamma_{5} \partial_{\mu} K_{c}^{\dagger} \tau \Xi+\bar{\Xi} \gamma^{\mu} \gamma_{5} \tau \partial_{\mu} K_{c} \cdot \Sigma\right] \\
& -f_{N N \eta_{8}} \bar{N} \gamma^{\mu} \gamma_{5} N \partial_{\mu} \eta \\
& -f_{\Lambda \Lambda \eta_{8}} \bar{\Lambda} \gamma^{\mu} \gamma_{5} \Lambda \partial_{\mu} \eta-f_{\Sigma \Sigma \eta_{8}} \bar{\Sigma} \cdot \gamma^{\mu} \gamma_{5} \Sigma \partial_{\mu} \eta \\
& -f_{\Xi \Xi \eta_{8}} \bar{\Xi} \gamma^{\mu} \gamma_{5} \Xi \partial_{\mu} \eta .
\end{aligned}
$$

The interaction Lagrangian in Eq. (4) is invariant under $\mathrm{SU}(3)_{\mathrm{f}}$ transformations if the various coupling constants fulfill specific relations which can be expressed in terms of the coupling constant $f$ and the $F /(F+D)$-ratio $\alpha$ as [18],

$$
\begin{aligned}
f_{N N \pi} & =f, & f_{N N \eta_{8}} & =\frac{1}{\sqrt{3}}(4 \alpha-1) f, \\
f_{\Lambda N K} & =-\frac{1}{\sqrt{3}}(1+2 \alpha) f, & f_{\Xi \Xi \pi} & =-(1-2 \alpha) f, \\
f_{\Xi \Xi \eta_{8}} & =-\frac{1}{\sqrt{3}}(1+2 \alpha) f, & f_{\Xi \Lambda K} & =\frac{1}{\sqrt{3}}(4 \alpha-1) f, \\
f_{\Lambda \Sigma \pi} & =\frac{2}{\sqrt{3}}(1-\alpha) f, & f_{\Sigma \Sigma \eta_{8}} & =\frac{2}{\sqrt{3}}(1-\alpha) f, \\
f_{\Sigma N K} & =(1-2 \alpha) f, & f_{\Sigma \Sigma \pi} & =2 \alpha f, \\
f_{\Lambda \Lambda \eta_{8}} & =-\frac{2}{\sqrt{3}}(1-\alpha) f, & f_{\Xi \Sigma K} & =-f .
\end{aligned}
$$

Here $f \equiv g_{A} / 2 F_{\pi}, g_{A}$ is the axial-vector strength, $g_{A}=$ 1.26 , which is measured in neutron $\beta$-decay and $F_{\pi}$ is the weak pion decay constant, $F_{\pi}=92.4 \mathrm{MeV}$. For the $F /(F+$ $D)$-ratio we adopt here the SU(6) value ( $\alpha=0.4)$ which was already used in our study of the $Y N$ system [13].

The spin-space part of the LO one-pseudoscalar-mesonexchange potential is similar to the static one-pion-exchange potential in [20] (recoil and relativistic corrections give higher order contributions),

$$
V^{O B E}=-f_{B_{1} B_{1}^{\prime} P} f_{B_{2} B_{2}^{\prime} P} \frac{\left(\sigma_{1} \cdot \mathbf{q}\right)\left(\boldsymbol{\sigma}_{2} \cdot \mathbf{q}\right)}{\mathbf{q}^{2}+m_{P}^{2}},
$$

where $m_{P}$ is the mass of the exchanged pseudoscalar meson. The transferred and average momentum, $\mathbf{q}$ and $\mathbf{k}$, are defined in terms of the final and initial center-of-mass (c.m.) momenta of the baryons, $\mathbf{p}^{\prime}$ and $\mathbf{p}$, as $\mathbf{q}=\mathbf{p}^{\prime}-\mathbf{p}$ and $\mathbf{k}=\left(\mathbf{p}^{\prime}+\mathbf{p}\right) / 2$. Note that we use the physical masses of the exchanged pseudoscalar mesons. Thus, the explicit SU(3) breaking reflected in the mass splitting between the pseudoscalar mesons is taken into account. The $\eta$ meson was identified with the octet $\eta\left(\eta_{8}\right)$ and its physical mass was used.

The reaction amplitudes are obtained from the solution of a coupled-channels Lippmann-Schwinger (LS) equation for the interaction potentials:

$$
\begin{aligned}
& T_{\rho^{\prime \prime} \rho^{\prime}}^{\nu^{\prime \prime} v^{\prime}, J}\left(p^{\prime \prime}, p^{\prime} ; \sqrt{s}\right)=V_{\rho^{\prime \prime} \rho^{\prime}}^{\nu^{\prime \prime} v^{\prime}, J}\left(p^{\prime \prime}, p^{\prime}\right)+ \\
& \sum_{\rho, v} \int_{0}^{\infty} \frac{d p p^{2}}{(2 \pi)^{3}} V_{\rho^{\prime \prime} \rho}^{v^{\prime \prime} v, J}\left(p^{\prime \prime}, p\right) \frac{2 \mu_{v}}{q_{\nu}^{2}-p^{2}+i \eta} T_{\rho \rho^{\prime}}^{\nu v^{\prime}, J}\left(p, p^{\prime} ; \sqrt{s}\right) .
\end{aligned}
$$

The label $v$ indicates the particle channels and the label $\rho$ the partial wave. $\mu_{v}$ is the pertinent reduced mass. The onshell momentum in the intermediate state, $q_{v}$, is defined by $\sqrt{s}=\sqrt{M_{B_{1, v}}^{2}+q_{v}^{2}}+\sqrt{M_{B_{2, v}}^{2}+q_{v}^{2}}$. Relativistic kinematics is used for relating the laboratory energy $T_{\text {lab }}$ of the hyperons to the c.m. momentum.

We solve the LS equation in the particle basis, in order to incorporate the correct physical thresholds. Depending
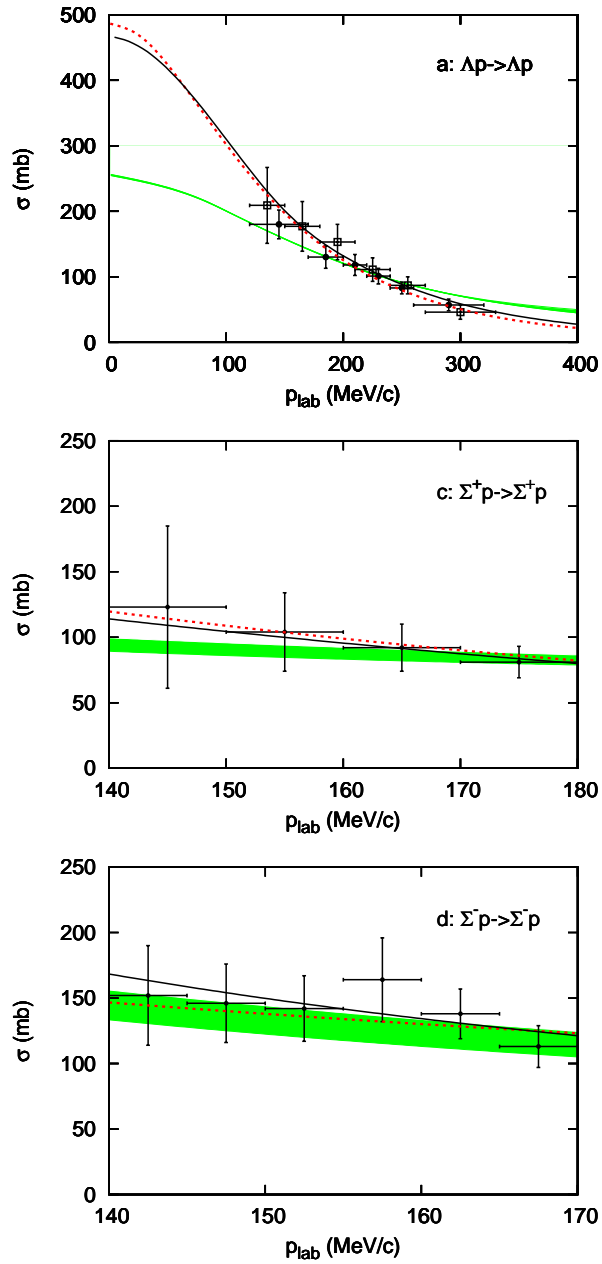

Fig. 1. $Y N$ integrated cross sections as a function of $p_{\text {lab. }}$ The band are results based on chiral EFT to LO for cutoffs $\Lambda=$ $550, \ldots, 700 \mathrm{MeV}$, while the solid and dashed curves are those of the Jülich '04 [21] and Nijmegen NSC97f [22] meson-exchange models, respectively.

on the specific values of strangeness and charge up to six baryon-baryon channels can couple. For the $S=-1$ sector where a comparison with scattering data is possible the Coulomb interaction is taken into account appropriately. The potentials in the LS equation are cut off with a regulator function, $\exp \left[-\left(p^{\prime 4}+p^{4}\right) / \Lambda^{4}\right]$, in order to remove high-energy components of the baryon and pseudoscalar meson fields [7]. We consider cut-off values in the range $550, \ldots, 700 \mathrm{MeV}$, similar to what was used for chiral $N N$ potentials [7].

\section{Results for the strangeness $S=-1$ and $\mathrm{S}=-2$ sectors}

The imposed SU(3) flavor symmetry implies that only five of the six LECs contribute to the $Y N$ interaction, namely $C^{27}, C^{10}, C^{10^{*}}, C^{8_{s}}$, and $C^{8_{a}}$, cf. Table 1 . These five contact terms were determined in [13] by a fit to the $Y N$ scattering data. Since the $N N$ data cannot be described with a 
EPJ Web of Conferences

Table 2. ${ }_{\Lambda}^{3} \mathrm{H}$ binding energy and $\Lambda$ separation energies for ${ }_{\Lambda}^{4} \mathrm{H}$.

\begin{tabular}{|c|c|c|c|c|c|c|c|c|}
\hline & & \multicolumn{4}{|c|}{ EFT LO } & \multirow[t]{2}{*}{ Jülich '04 } & \multirow[t]{2}{*}{ NSC97f } & \multirow[t]{2}{*}{ expt. } \\
\hline & $\Lambda[\mathrm{MeV}]$ & 550 & 600 & 650 & 700 & & & \\
\hline${ }_{\Lambda}^{3} \mathrm{H}$ & $E_{B}[\mathrm{MeV}]$ & -2.35 & -2.34 & -2.34 & -2.36 & -2.27 & -2.30 & $-2.354(50)$ \\
\hline \multirow[t]{5}{*}{${ }_{\Lambda}^{4} \mathrm{H}$} & $E_{\text {sep }}\left(0^{+}\right)[\mathrm{MeV}]$ & 2.63 & 2.46 & 2.36 & 2.38 & 1.87 & 1.60 & 2.04 \\
\hline & $E_{\text {sep }}\left(1^{+}\right)[\mathrm{MeV}]$ & 1.85 & 1.51 & 1.23 & 1.04 & 2.34 & 0.54 & 1.00 \\
\hline & $\Delta E_{\text {sep }}[\mathrm{MeV}]$ & 0.78 & 0.95 & 1.13 & 1.34 & -0.48 & 0.99 & 1.05 \\
\hline & $\mathrm{CSB}^{-0^{+}}[\mathrm{MeV}]$ & 0.01 & 0.02 & 0.02 & 0.03 & -0.01 & 0.12 & 0.35 \\
\hline & $\mathrm{CSB}-1^{+}[\mathrm{MeV}]$ & -0.01 & -0.01 & -0.01 & -0.01 & - & -0.01 & 0.24 \\
\hline
\end{tabular}

LO EFT, SU(3) constraints from the $N N$ interaction were not implemented explicitly. As shown in Ref. [13], a good description of the 35 low-energy $Y N$ scattering can be obtained for cutoff values $\Lambda=550, \ldots, 700 \mathrm{MeV}$ and for natural values of the LECs. Some of the results are reproduced here in Fig. 1. The shaded band represents the variation of cross sections based on chiral EFT for the considered cutoff region. For comparison also results for the Jülich '04 [21] (solid line) and the Nijmegen NSC97f [22] (dashed line) meson-exchange models are shown.

Besides a reasonable description of the $Y N$ data our LO chiral EFT interaction also yields a correctly bound hypertriton, see Table 2. Exploratory calculations for the fourbody hypernuclei ${ }_{\Lambda}^{4} \mathrm{H}$ and ${ }_{\Lambda}^{4} \mathrm{He}$, reported in [23] and reproduced here in Table 2, suggest that the chiral EFT predicts reasonable $\Lambda$ separation energies for ${ }_{\Lambda}^{4} \mathrm{H}$, but the charge dependence of the $\Lambda$ separation energies is not reproduced. In the table the separation energies of the $0^{+}\left(E_{\text {sep }}\left(0^{+}\right)\right)$and $1^{+}\left(E_{\text {sep }}\left(1^{+}\right)\right)$states for ${ }_{\Lambda}^{4} \mathrm{H}$ as well as the difference of the separation energies for the mirror hypernuclei ${ }_{\Lambda}^{4} \mathrm{He}$ and ${ }_{\Lambda}^{4} \mathrm{H}$ $\left(\mathrm{CSB}-0^{+}\right.$and $\left.\mathrm{CSB}-1^{+}\right)$are given.

The sixth LEC $\left(C^{1}\right)$ is only present in the $S=-2$ channels with isospin zero, cf. Table 1 . There is scarce experimental information on these channels [24] that could be used to fix this LEC, but it turned out that the quality of the existing data do not really allow to constrain its value reliably [14]. Our results for the $S=-2$ sector are shown in Figs. 2 and 3. The bands reflect the dependence of the results on variations of the cutoff $\Lambda$. The cutoff was varied between 550 and $700 \mathrm{MeV}$ (like in case of $Y N$ ) and under the constraint that the $\Lambda \Lambda{ }^{1} S_{0}$ scattering length remains practically unchanged [14]. As reference we have taken the result for $\Lambda=600 \mathrm{MeV}$ and with the value of the sixth LEC set to zero. This yields a scattering length of $a_{1}^{\Lambda \Lambda}=-1.52 \mathrm{fm}$ [14]. Obviously, the chiral EFT results are consistent with the empirical cross sections for such variations. Note that the results in Fig. 3 are independent of the sixth LEC because they involve only isospin 1 or 2 amplitudes and, thus, genuine predictions based on our fit to the $Y N$ data.
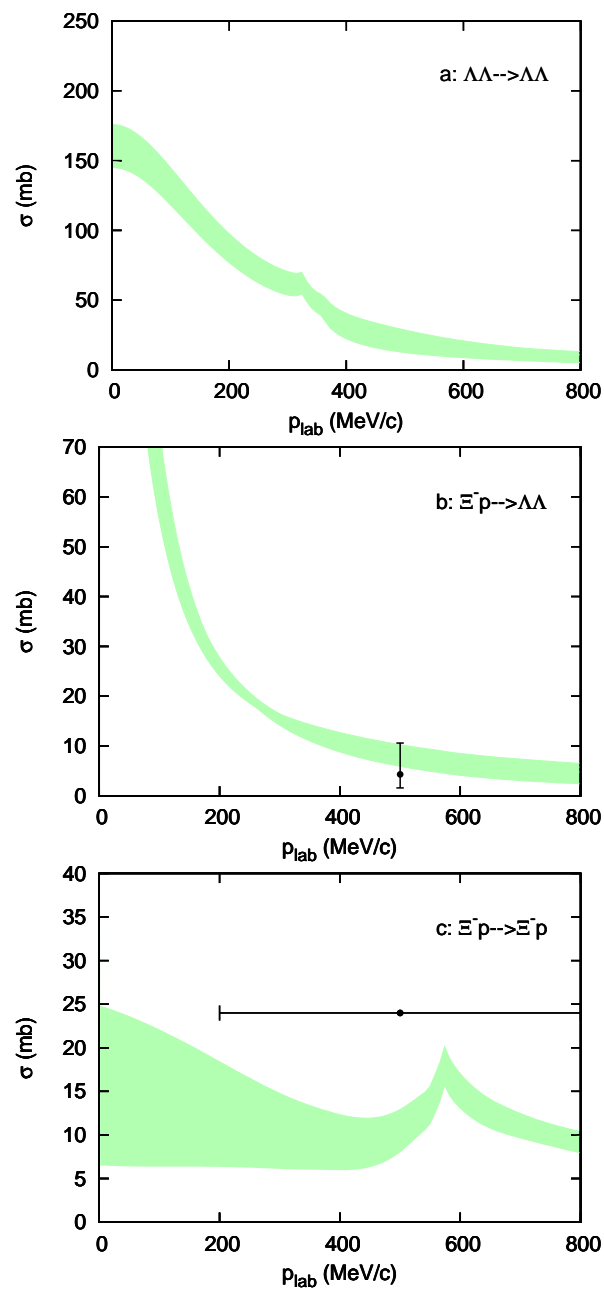

Fig. 2. $Y Y$ and $\Xi N$ integrated cross sections as a function of $p_{\text {lab. }}$. The band shows the chiral EFT for variations of the cutoff $\Lambda$ as discussed in the text. Data are from Ref. [24].

\section{Results for the strangeness $S=-3$ and $\mathrm{S}=-4$ sectors}

The LO chiral EFT interaction for the $S=-3$ and -4 baryon-baryon sector depends only on those five contact terms that enter also in the $Y N$ interaction, as can be seen from Table 1. Thus, based on the values which were fixed in our study of the $Y N$ sector [13] we can make genuine 
$19^{\text {th }}$ International IUPAP Conference on Few-Body Problems in Physics
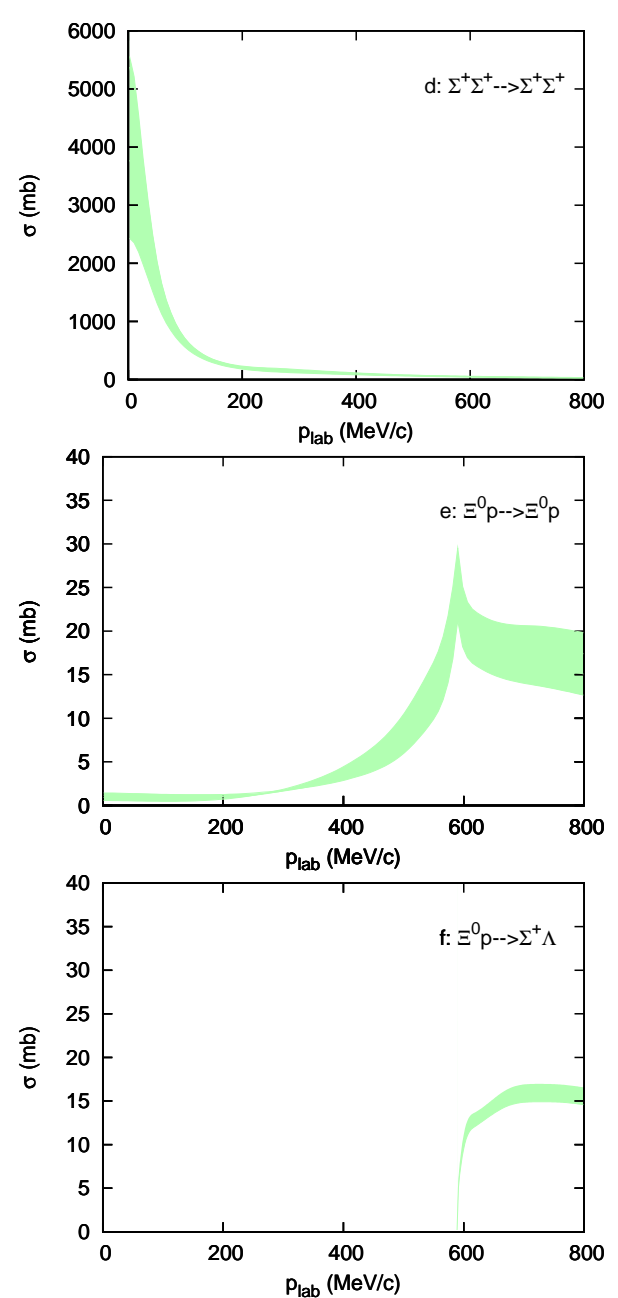

Fig. 3. $Y Y$ and $\Xi N$ integrated cross sections as a function of $p_{\text {lab }}$. The band shows the chiral EFT for variations of the cutoff $\Lambda$ as discussed in the text.

predictions for the interaction in the $S=-3$ and -4 channels that follow from the imposed $\mathrm{SU}(3)_{\mathrm{f}}$ symmetry.

Corresponding results for the $\Xi^{0} \Lambda \rightarrow \Xi^{0} \Lambda, \Xi^{0} \Sigma^{-} \rightarrow$ $\Xi^{-} \Lambda, \Xi^{0} \Sigma^{-} \rightarrow \Xi^{-} \Sigma^{0}, \Xi^{0} \Sigma^{-} \rightarrow \Xi^{0} \Sigma^{-}$, and $\Xi^{0} \Sigma^{+} \rightarrow \Xi^{0} \Sigma^{+}$ scattering cross sections are presented in Fig. 4. Partial waves with total angular momentum up-to-and-including $J=2$ are taken into account. The shaded bands show the cut-off dependence. From that figure one observes that the $\Xi^{0} \Lambda \rightarrow \Xi^{0} \Lambda$ and $\Xi^{0} \Sigma^{+} \rightarrow \Xi^{0} \Sigma^{+}$cross sections are rather large near threshold. Though the cross section for $\Xi^{0} \Sigma^{-} \rightarrow \Xi^{-} \Lambda$ rises too, in this case it is only due to the phase space factor $p_{\Xi^{-} \Lambda} / p_{\Xi^{0} \Sigma^{-}}$. There is a clear cusp effect visible in the $\Xi^{0} \Sigma^{-}$cross section at $p_{\text {lab }} \approx 106 \mathrm{MeV} / \mathrm{c}$, i.e. at the opening of the $\Xi^{-} \Sigma^{0}$ channel. On the other hand, we do not observe any sizeable cusp effects in the $\Xi^{0} \Lambda$ cross section around $p_{\text {lab }}=690 \mathrm{MeV} / \mathrm{c}$, i.e. at the opening of the $\Xi \Sigma$ channels. The latter is in line with the results reported by the Nijmegen group for their interactions [25], where a cusp effect in that channel is absent too. In this context I would like to remind the reader that the cusp seen in the corresponding strangeness $S=-1$ case, namely in the $\Lambda N$ cross section at the $\Sigma N$ threshold, is rather pronounced
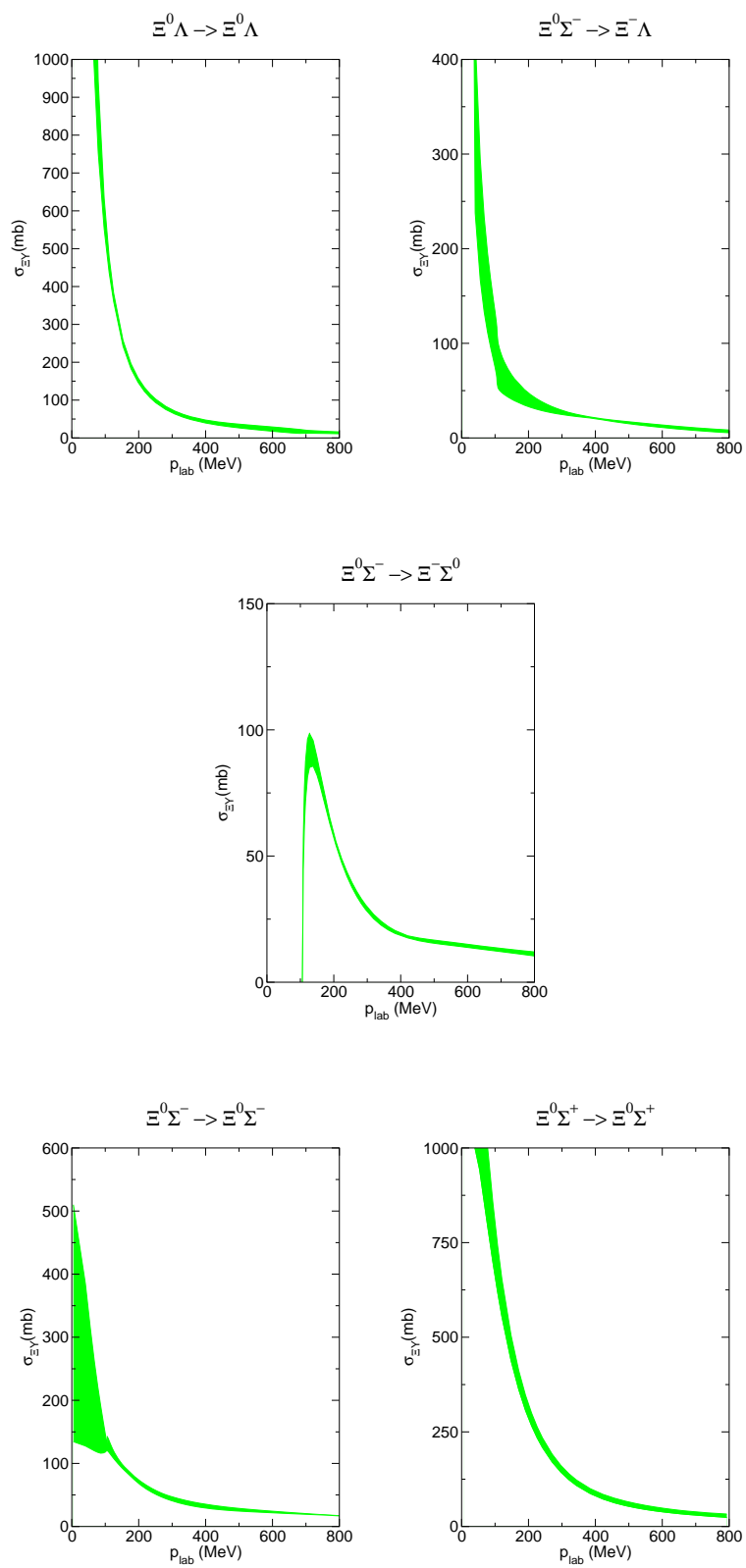

Fig. 4. Total cross sections for various reactions in the strangeness $S=-3$ sector as a function of $p_{\text {lab }}$. The shaded band shows the chiral EFT results for variations of the cut-off in the range $\Lambda=$ 550. . .700 MeV.

in our chiral EFT interaction [13] but also in conventional meson-exchange potential models [21,22].

Predicted cross sections for the $\Xi^{0} \Xi^{0}$ and $\Xi^{0} \Xi^{-}$channels are shown in Fig. 5, again as a function of $p_{l a b}$ and with shaded bands that indicate the cut-off dependence.

Results for the $\Xi^{0} \Lambda, \Xi^{0} \Sigma^{+}$, and $\Xi \Xi$ scattering lengths and effective ranges are listed in Table 3 . Here we also include predictions by other models $[25,26]$ for channels where pertinent results are available in the literature. This Table reveals the reason for the sizeable $\Xi^{0} \Lambda$ cross sec- 
Table 3. Selected $\Xi Y$ and $\Xi \Xi$ singlet and triplet scattering lengths $a$ and effective ranges $r$ (in fm) for various cut-off values $\Lambda$. The last columns show results for the Nijmegen potential (NSC97a, NSC97f) [25] and the model by Fujiwara et al. (fss2) [26].

\begin{tabular}{|c|c|c|c|c|c|c|c|}
\hline & \multicolumn{4}{|c|}{ EFT } & NSC97a & NSC97f & fss 2 \\
\hline$\Lambda(\mathrm{MeV})$ & 550 & 600 & 650 & 700 & & & \\
\hline$a_{s}^{\Xi \Lambda}$ & -33.5 & 35.4 & 12.7 & 9.07 & -0.80 & -2.11 & -1.08 \\
\hline$r_{s}^{ \pm \Lambda}$ & 1.00 & 0.93 & 0.87 & 0.84 & 4.71 & 3.21 & 3.55 \\
\hline$a_{t}^{\Xi \Lambda}$ & 0.33 & 0.33 & 0.32 & 0.31 & 0.54 & 0.33 & 0.26 \\
\hline$r_{t}^{\Xi \Lambda}$ & -0.36 & -0.30 & -0.29 & -0.27 & -0.47 & 2.79 & 2.15 \\
\hline$a_{s}^{\varepsilon^{0} \Sigma^{+}}$ & 4.28 & 3.45 & 2.97 & 2.74 & 4.13 & 2.32 & -4.63 \\
\hline$r_{s}^{z^{0} \Sigma^{+}}$ & 0.96 & 0.90 & 0.84 & 0.81 & 1.46 & 1.17 & 2.39 \\
\hline$a_{t}^{\Xi^{0} \Sigma^{+}}$ & -2.45 & -3.11 & -3.57 & -3.89 & 3.21 & 1.71 & -3.48 \\
\hline$r_{t}^{\Xi^{0} \Sigma^{+}}$ & 1.84 & 1.72 & 1.70 & 1.70 & 1.28 & 0.96 & 2.52 \\
\hline 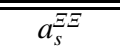 & 3.92 & 3.16 & 2.71 & 2.47 & 17.28 & 2.38 & $\overline{-1.43}$ \\
\hline$r_{s}^{s} \Xi$ & 0.92 & 0.85 & 0.79 & 0.75 & 1.85 & 1.29 & 3.20 \\
\hline$a_{t}^{\Xi \Xi}$ & 0.63 & 0.59 & 0.55 & 0.52 & 0.40 & 0.48 & 3.20 \\
\hline$r_{t}^{E \Xi}$ & 1.04 & 1.05 & 1.08 & 1.11 & 3.45 & 2.80 & 0.22 \\
\hline
\end{tabular}
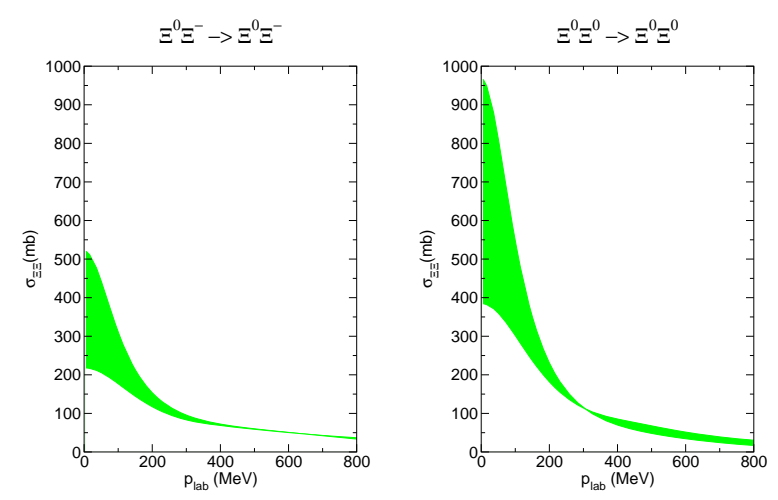

Fig. 5. Total cross sections for the reactions $\Xi^{0} \Xi^{-} \rightarrow \Xi^{0} \Xi^{-}$and $\Xi^{0} \Xi^{0} \rightarrow \Xi^{0} \Xi^{0}$ as a function of $p_{l a b}$. The shaded band shows the chiral EFT results for variations of the cut-off in the range $\Lambda=$ $550 . .700 \mathrm{MeV}$.

tion predicted by the chiral EFT interactions, namely a rather large scattering length in the corresponding ${ }^{1} S_{0}$ partial wave. It is obvious that its value is strongly sensitive to cut-off variations. It even changes sign (in other words, it becomes infinite) within the considered cut-off range. This means that a virtual bound state transforms into a real bound state, where the strongest binding occurs for the cut-off $\Lambda=700 \mathrm{MeV}$ and leads to a binding energy of $-0.43 \mathrm{MeV}$. While this behaviour is interesting per se, one certainly has to stress that in such a case the predictive power of our LO calculation is rather limited. One has to wait for at least an NLO calculation, where we expect that the cut-off dependence will become much weaker so that more reliable conclusions on the possible existence of a virtual or a real bound state should be possible. The ${ }^{1} S_{0}$ scattering lengths of the other potentials suggest also an overall attractive interaction in this partial wave though only a very moderate one.
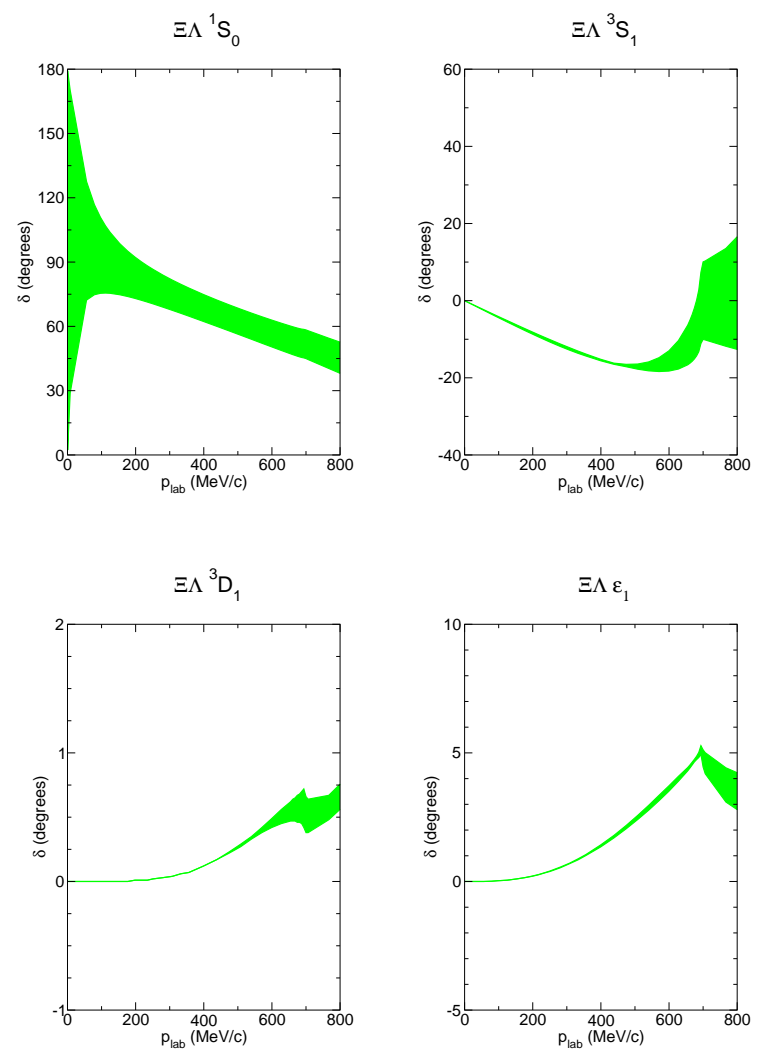

Fig. 6. Selected phase shifts for $\Xi \Lambda$. The shaded band shows the chiral EFT results to $\mathrm{LO}$ for variations of the cut-off in the range $\Lambda=550 \ldots .700 \mathrm{MeV}$

The results for the ${ }^{3} S_{1}$ state of the $\Xi^{0} \Lambda$ channel are fairly similar for all considered interactions. Moreover, with regard to the chiral EFT interaction there is very little cutoff dependence. The $S$-waves in the $\Xi \Sigma I=3 / 2$ channel belong to the same ( $10^{*}$ and 27 , respectively) irreducible 

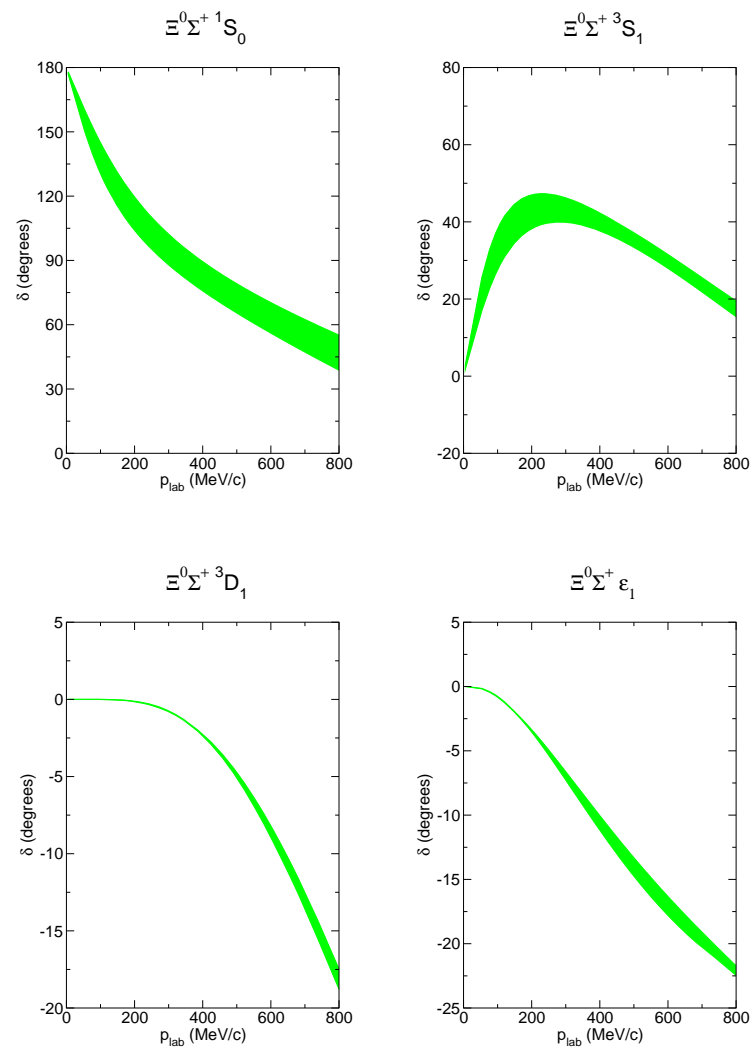

Fig. 7. Selected phase shifts for $\Xi^{0} \Sigma^{+}$. The shaded band shows the chiral EFT results to LO for variations of the cut-off in the range $\Lambda=550 . .700 \mathrm{MeV}$

representations where in the $N N$ case real $\left({ }^{3} S_{1}-{ }^{3} D_{1}\right)$ or virtual $\left({ }^{1} S_{0}\right)$ bound states exist, cf. Table 1 in Ref. [15]. Therefore, one expects that such states can also occur for $\Xi \Sigma$. Indeed, bound states are present for both partial waves in the Nijmegen model, cf. the discussion in Sect. III.B in Ref. [25]. Their presence is reflected in the positive and fairly large singlet and triplet scattering lengths for $\Xi^{0} \Sigma^{+}$, cf. Table 3. The chiral EFT interaction has positive scattering lengths of comparable magnitude for ${ }^{1} S_{0}$, for all cutoff values, and therefore bound states, too. These binding energies lie in the range of $-2.23 \mathrm{MeV}(\Lambda=550 \mathrm{MeV})$ to $-6.15 \mathrm{MeV}(700 \mathrm{MeV})$. In the ${ }^{3} S_{1}-{ }^{3} D_{1}$ partial wave the attraction is obviously not strong enough to form a bound state. The same is the case (but for both $S$ waves) for the quark model fss2 of Fujiwara et al. [26].

The ${ }^{1} S_{0}$ state of the $\Xi \Xi$ channel belongs also to the 27plet irreducible representation and also here the $\mathrm{Ni}$ jmegen as well as the chiral EFT interactions produce bound states. In our case the binding energies lie in the range of $-2.56 \mathrm{MeV}(\Lambda=550 \mathrm{MeV})$ to $-7.28 \mathrm{MeV}(700$ $\mathrm{MeV})$. The predictions of both approaches for the ${ }^{3} S_{1}$ scattering length are comparable. The quark model of Fujiwara et al. exhibits a different behavior for the $\Xi \Xi$ channel, see the last column in Table 3 . The small and negative ${ }^{1} S_{0}$ scattering length signals an interaction that is only moderately
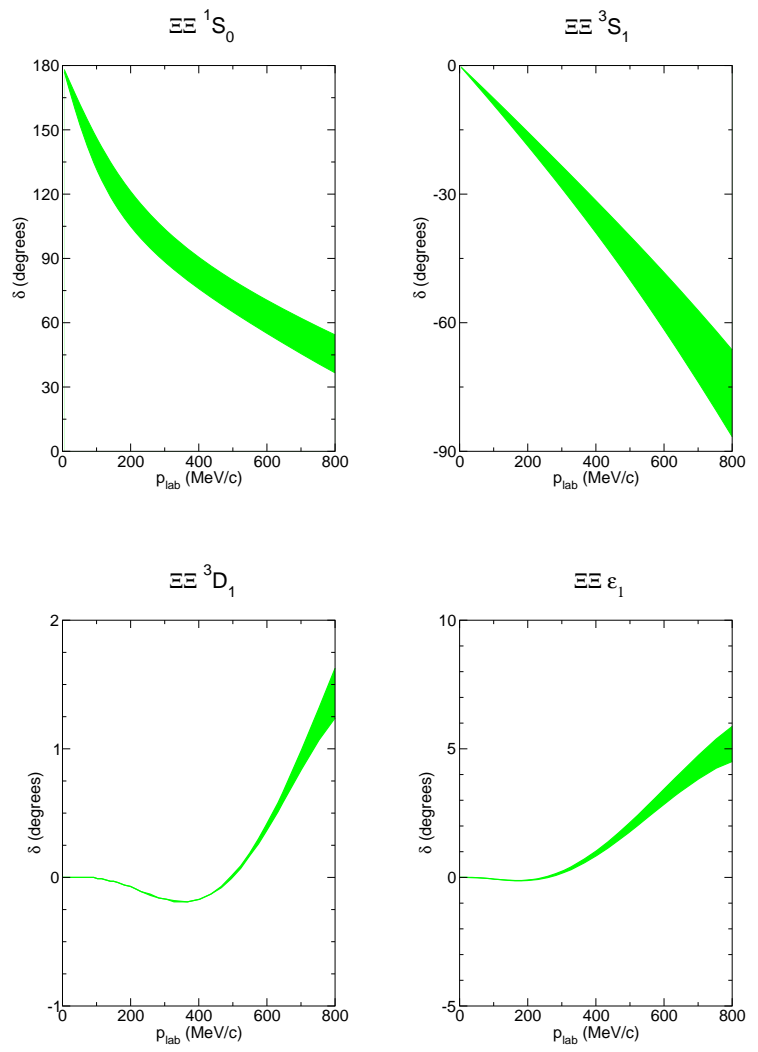

Fig. 8. Selected phase shifts for $\Xi \Xi$. The shaded band shows the chiral EFT results to $\mathrm{LO}$ for variations of the cut-off in the range $\Lambda=550 \ldots .700 \mathrm{MeV}$

attractive. The large and positive scattering length in the ${ }^{3} S_{1}-{ }^{3} D_{1}$ partial wave, produced by that potential model, is usually a sign for the presence of a bound state, though according to the authors this is not the case for this specific interaction.

Finally, let me show here predictions for some selected $\Xi \Lambda, \Xi \Sigma$ and $\Xi \Xi$ phase shifts. Corresponding results for the ${ }^{1} S_{0}$ and the coupled $3 S_{1}-{ }^{3} D_{1}$ partial waves for $\Xi \Lambda$ are depicted in Fig. 6. The large uncertainty seen in the $1 S_{0}$ case near threshold reflects the large variations in the scattering lengths, discussed already above. Note that the phase shifts start at 180 or zero degrees depending on whether a bound state is present or not, in accordance with Levinson's theorem. In the triplet partial waves one sees clearly the opening of the $\Xi \Sigma$ threshold at around $690 \mathrm{MeV} / \mathrm{c}$. However, the effect is here less pronounced than at the corresponding $\Lambda N$ case at the $\Sigma N$ threshold, cf. Fig. 7 in [13].

The ${ }^{1} S_{0}$ partial waves of $\Xi^{0} \Sigma^{+}$and $\Xi \Xi$ belong to the same (27) irreducible representation and, moreover, both do not couple to any other baryon-baryon channel. The predicted phase shifts are indeed very similar as one would expect from the imposed SU(3) symmetry, cf. Figs. 7 and 8 . The same is the case for the ${ }^{3} S_{1}$ phase shift for the $\Xi \Xi$ channel, shown in Fig. 8 and the corresponding one for $\Sigma^{+} p$, which can be found in Fig. 9 of [13], which both belong to the (10) irreducible representation. 

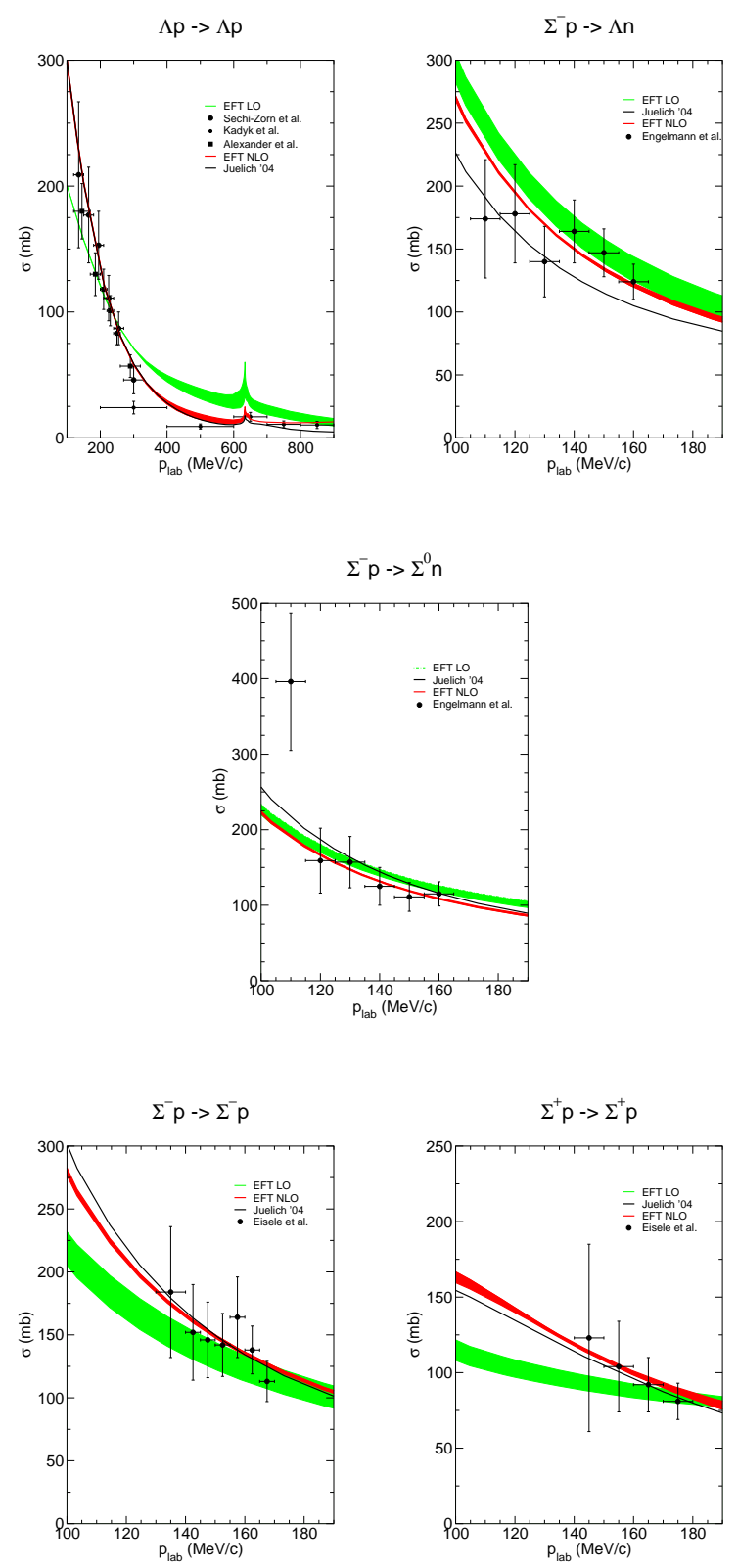

Fig. 9. Total cross sections for $\Lambda p \rightarrow \Lambda p, \Sigma^{-} p \rightarrow \Lambda n, \Sigma^{-} p \rightarrow$ $\Sigma^{0} n, \Sigma^{-} p \rightarrow \Sigma^{-} p$ and $\Sigma^{+} p \rightarrow \Sigma^{+} p$ as a function of $p_{\text {lab }}$. The green (grey) band shows the chiral EFT results to LO for variations of the cut-off in the range $\Lambda=550 \ldots 700 \mathrm{MeV}$, while the red (black) band are preliminary results to NLO. The solid curve is the result of the Jülich '04 [21] meson-exchange potential.

\section{Extension to next-to-leading order}

In next-to-leading order one gets contributions from (noniterative) two-pseudoscalar-meson exchange diagrams [7, 16]. In addition there are four-baryon contact terms with two derivatives. The spin- and momentum structure of the latter is given by

$$
V^{(2)}=C_{1} \mathbf{q}^{2}+C_{2} \mathbf{k}^{2}+\left(C_{3} \mathbf{q}^{2}+C_{4} \mathbf{k}^{2}\right)\left(\sigma_{1} \cdot \sigma_{2}\right)
$$

$$
\begin{aligned}
& +i C_{5}\left(\sigma_{1}+\sigma_{2}\right) \cdot(\mathbf{q} \times \mathbf{k})+C_{6}\left(\mathbf{q} \cdot \sigma_{1}\right)\left(\mathbf{q} \cdot \sigma_{2}\right) \\
& +C_{7}\left(\mathbf{k} \cdot \sigma_{1}\right)\left(\mathbf{k} \cdot \sigma_{2}\right)+i C_{8}\left(\sigma_{1}-\sigma_{2}\right) \cdot(\mathbf{q} \times \mathbf{k})
\end{aligned}
$$

The $C_{i}$ 's are additional LECs. Performing a partial wave projection and imposing again $\mathrm{SU}(3)_{\mathrm{f}}$ symmetry one finds that in case of the $Y N$ interaction there are ten new coefficents entering the $S$ waves and $S-D$ transitions, respectively, and eight coefficents in the $P$ waves. All of those need to be determined by a fit to data.

In Fig. 9 some preliminary results are given in order to illustrate what can be achieved within NLO. I would like to emphasize, however, that these calculations are still incomplete. So far only the additional contact terms in $S$ waves are taken into account and not the ones contributing to the $P$-waves. Furthermore, all two-pseudoscalar-meson exchange diagrams are missing. As already done in case of $\mathrm{LO}$, no $\mathrm{SU}(3)_{\mathrm{f}}$ constraints from the $N N$ sector are imposed here and also the leading order $\mathrm{SU}(3)_{\mathrm{f}}$ breaking in the oneboson exchange diagrams (coupling constants) is ignored. Work on completing the NLO calculation is in progress [16].

Obviously, and as expected, the energy dependence exhibited by the data can be significantly better reproduced within our NLO calculation. This concerns in particular the $\Sigma^{+} p$ channel. But also for $\Lambda p$ the NLO results are now well in line with the data even up to the $\Sigma N$ threshold. Furthermore, one can see that the dependence on the cutoff mass is strongly reduced in the NLO case.

\section{Summary}

Our findings show that the chiral EFT scheme, successfully applied in Ref. [7] to the $N N$ interaction, also works well for the $\Lambda N, \Sigma N$ and $\Lambda \Lambda$ interactions. Moreover, it can be used o make predictions for the $S=-3$ and -4 baryonbaryon interactions invoking constraints from $\mathrm{SU}(3)$ flavor symmetry. It turned out that already at LO the bulk properties of the $\Lambda N$ and $\Sigma N$ systems can be reasonably well accounted for. Our preliminary and still incomplete results for the $Y N$ interaction to next-to-leading order look very promising. It will be interesting to see what can be achieved within a full calculation to NLO. Work in this direction is in progress.

I would like to thank Evgeny Epelbaum, Simon Kazmierowski, Ulf-G. Meißner, Andreas Nogga, and Henk Polinder for collaborating on the topic covered by my talk.

\section{References}

1. S. Weinberg, Phys. Lett. B 251 (1990) 288.

2. S. Weinberg, Nucl. Phys. B 363 (1991) 3.

3. P. F. Bedaque, U. van Kolck, Annu. Rev. Nucl. Part. Sci. 52 (2002) 339.

4. E. Epelbaum, Prog. Part. Nucl. Phys. 57 (2006) 654.

5. E. Epelbaum, H. W. Hammer and U.-G. Meißner, Rev. Mod. Phys., in print, arXiv:0811.1338 [nucl-th]. 
6. D. R. Entem, R. Machleidt, Phys. Rev. C 68 (2003) 041001.

7. E. Epelbaum, W. Glöckle, U.-G. Meißner, Nucl. Phys. A 747 (2005) 362.

8. M. J. Savage, M. B. Wise, Phys. Rev. D 53 (1996) 349.

9. H. W. Hammer, Nucl. Phys. A705 (2002) 173.

10. C. L. Korpa, A. E. L. Dieperink, R. G. E. Timmermans, Phys. Rev. C 65 (2001) 015208.

11. D. B. Kaplan, M. J. Savage, M. B. Wise, Nucl. Phys. B534 (1998) 329.

12. S. R. Beane, P. F. Bedaque, A. Parreño, M. J. Savage, Nucl. Phys. A747 (2005) 55.

13. H. Polinder, J. Haidenbauer and U.-G. Meißner, Nucl. Phys. A 779 (2006) 244.

14. H. Polinder, J. Haidenbauer and U.-G. Meißner, Phys. Lett. B 653 (2007) 29.

15. J. Haidenbauer, U.-G. Meißner, arXiv:0907.1395 [nucl-th].

16. S. Kazmierowski, diploma thesis, University of Bonn, 2009.

17. J. Haidenbauer, U.-G. Meißner, A. Nogga and H. Polinder, Lect. Notes Phys. 724 (2007) 113.

18. J. J. de Swart, Rev. Mod. Phys. 35 (1963) 916.

19. C. B. Dover and H. Feshbach, Annals Phys. 217 (1992) 51.

20. E. Epelbaoum, W. Glöckle, and U.-G. Meißner, Nucl. Phys. A 637 (1998) 107.

21. J. Haidenbauer, U.-G. Meißner, Phys. Rev. C 72 (2005) 044005.

22. T. A. Rijken, V. G. J. Stoks, Y. Yamamoto, Phys. Rev. C 59 (1999) 21.

23. A. Nogga, arXiv:nucl-th/0611081.

24. J.K. Ahn et al., Phys. Lett. B 633 (2006) 214.

25. V. G. J. Stoks and T. A. Rijken, Phys. Rev. C 59 (1999) 3009.

26. Y. Fujiwara, Y. Suzuki and C. Nakamoto, Prog. Part. Nucl. Phys. 58 (2007) 439. 